\title{
Deformity prevention in the field: a systematic approach
}

\author{
JEAN HAMILTON \\ The Leprosy Mission Hospital, Salur, Andhra Pradesh, South \\ India*
}

Accepted for publication 24 March 1983

\begin{abstract}
Summary The importance of preventing deformity among our patients by detecting and treating neuritis is stated. The process of 'silent neuritis' which leads insidiously to deformity in a number of leprosy patients is defined, and the importance of its recognition and early treatment in the field situation is stressed. An approach adopted primarily for this purpose, consisting of simple motor, sensory and nerve palpation tests repeated at varying intervals, whose results are recorded briefly and acted upon appropriately, is described. The benefits observed and problems encountered in implementing this approach are described, and areas of training and management on which its success depends are discussed.
\end{abstract}

\section{Introduction}

Prevention or reduction of deformity is important for the personal, economic and emotional welfare of leprosy patients. It may contribute to the effectiveness of the leprosy control programme, and also be of value in changing the public image of leprosy. It has been well stated that 'the control of leprosy depends as much on the effective prevention and treatment of disability (which is the patient's primary concern) as on the reduction of the mycobacterial load in the community by chemotherapy'.1

\section{CAUSE OF DEFORMITY}

The deformities considered in this paper are those resulting from nerve damage. Loss of sensory and/or motor function leads initially to anaesthesia and/or muscle paralysis in the area supplied by the nerve, and subsequently, if neglected, to tissue injuries and joint stiffness, these being the visible and stigmatizing evidence of leprosy. Such nerve damage occurs in two particular phases of the

* Present address: 2 Horseleys Park, St Andrews, Fife KY16 8RZ. 
disease, ${ }^{2}$ namely in untreated leprosy, and during the course of both Type I and Type II reactions. Many deformities will therefore be prevented by early diagnosis of leprosy, followed by regular and adequate treatment with antileprosy drugs, but in the presence of reaction, treated patients remain at risk of developing deformity. Also women may be at special risk of nerve damage during pregnancy and lactation, ${ }^{3}$ when they may develop new deformity in the absence of reaction. We are therefore convinced that special care must be given to patients registered at our clinics, to minimize deformity occurring during reaction, pregnancy, etc.

\section{MODE OF ONSET}

Nerve damage caused by neuritis may present in either acute or silent forms. 'Silent' neuritis is defined by us as the presence of recent motor or sensory loss unaccompanied by nerve pain, of which the patient is usually unaware, and therefore does not complain. It closely resembles 'quiet nerve paralysis' as recently described by Srinivasan et al. from India. ${ }^{4}$ By contrast, 'acute' neuritis, elsewhere described as 'overt', ${ }^{3}$ is characterized by nerve pain and/or sudden, severe loss of function, which causes the patient variable but definite distress, and is therefore usually reported before deformity is established.

When recognized and treated early, a good proportion of neuritis patients show recovery, or limitation of nerve damage. In our experience, patients with acute neuritis, being easily recognized, are usually treated, whereas silent neuritis may go unnoticed and untreated, and lead to new deformity. Among lactating women, silent neuritis has been found to occur more than overt, ${ }^{3}$ highlighting this insidious problem, which, however, is not confined to women or to the period following childbirth.

It has also been observed that there is a large 'intermediate' group of patients who have mild neuritis clinically, and who complain of limb pain, who do not actually rate as having a reaction. These too may go on to develop permanent nerve damage, in the absence of appropriate neuritis treatment. The physiotherapy worker, hereafter referred to as physiotherapist, is traditionally associated with deformity care. The government pattern of leprosy control in India now includes a physiotherapist in the field team, and many voluntary agencies do likewise. However, these workers are often totally preoccupied with visible, established deformity about which comparatively little can be done, to the exclusion of concern to prevent new deformities occurring in apparently normal patients. Reversal and adaption of the philosophy 'innocent until proved guilty' to 'potentially deformed until proved normal' has much to commend it in the search for cases of silent neuritis. The method of assessment and its application, as now described, has been developed during the past 5 years in the leprosy control programme of the Leprosy Mission Hospital at Salur, South India, in order primarily to detect such cases. 


\section{Method}

Rapid but comprehensive assessment of the patient provides a baseline on nerve function. This can be used first to detect changes occurring, so diagnosing silent neuritis early, and secondly to guide the education and treatment required for existing deformity.

\section{METHOD OF ASSESSMENT}

\section{(a) Nerve palpation}

The ulnar, median, lateral popliteal and posterior tibial nerves of both arms and both legs are palpated at the sites of predilection. Nerve tenderness, ${ }^{5}$ recognized by watching the patient's face for a wincing expression, and enlargement, are noted.

Since nerve enlargement is a cardinal sign of leprosy, and it is difficult to detect further enlargement of already enlarged nerves - a sign of neuritis - significance is attached mainly to tenderness. This is almost always a sign of neuritis in a registered leprosy patient, though other diseases can cause nerve tenderness. ${ }^{1}$

\section{(b) Movement testing}

Closure of the eye-lids, opposition of thumbs to little fingers (or separate finger and thumb abduction) and ankle dorsiflexion are checked. Where range is full, resistance to movement is applied. Weakness is noted. A note on the Voluntary Muscle Test (VMT) grading of the muscle producing any movement found to be weak is added when the tester is a physiotherapist. This, when repeated at the next visit, provides more accurate information on the state of the nerve, and response to treatment given.

\section{(c) Sensory testing}

The cornea is tested carefully with a cotton wisp, but the palms and soles are tested for response to light pressure, using a ball-pen. Partial or complete anaesthesia is noted. In our opinion, this test gives the least accurate information regarding new nerve damage, but shows at an early stage which patients lack protective sensation and must therefore learn to prevent secondary ulceration.

A definite order of testing each patient is adopted, either doing tests for his eyes, then his hands, then his feet; or checking first his nerves, then his movements, and then sensation. Such a system is not always adopted automatically, but it increases both the speed of testing and the reliability of the results. 


\section{RECORDING OF FINDINGS}

A rubber stamp, covering relevant movements, sensation and nerves in the eyes, hands and feet, permits quick and accurate recording by a set of standard symbols, understood by all our team members. The deformity assessment is stamped in the patient's treatment chart beside the prescription of medicine and not separately, so that information is readily seen by all. Duration of movement weakness and sensory loss (if known) is recorded. Additional notes are written on points not covered by the stamp.

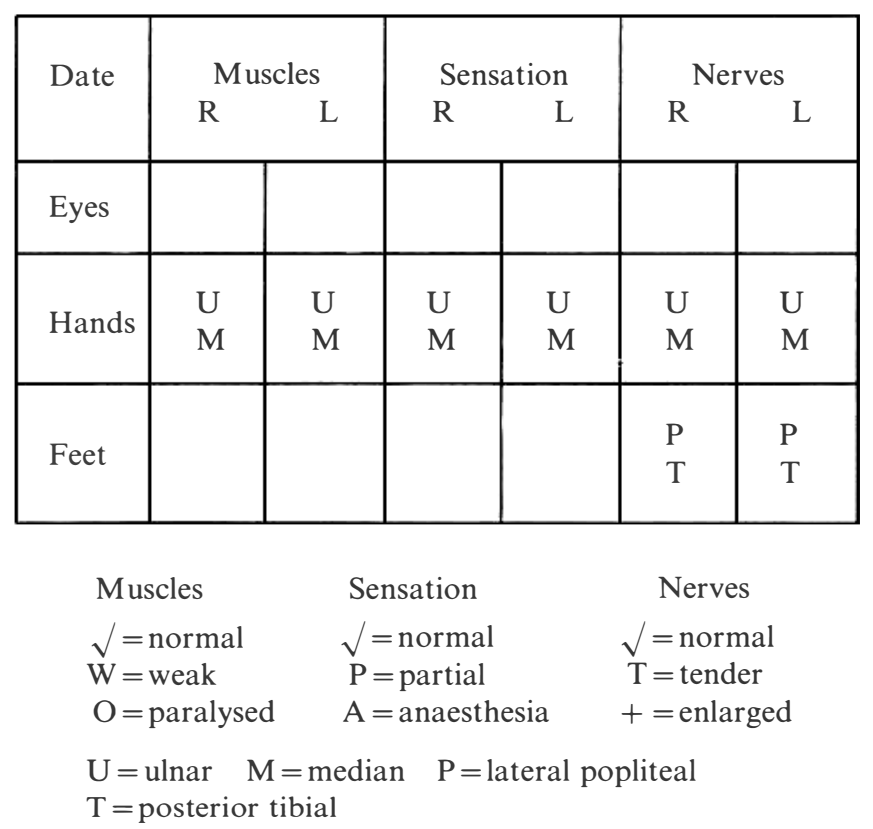

\section{FREQUENCY OF ASSESSMENT}

New patients are now assessed at the first attendance, and most previously registered patients have also been assessed. Thus all have baseline data.

Undeformed or partially deformed patients are rechecked as often as possible, but the suggested ideal of monthly, or at every clinic visit, is probably unnecessary and seldom practicable. Every effort, however, is made to check the following patients, considered to be most 'at risk' of neuritis and deformity, at least every 3 months: borderline cases, patients in their first year of treatment, lepromatous patients prone to recurrent Type II reactions, patients who have been irregular, and those who have had recent complications. Those with particular complaints 
are checked immediately. It must be stressed that annual reassessment will not detect silent neuritis in time for treatment to be effective.

\section{ACTION TO BE TAKEN ON FINDINGS}

(a) Patients with nerve tenderness, and/or movement weakness or sensory loss of recent duration (from patient's report, or by comparison with previous assessment) are given a warm wrapping for the nerve, and a rest sling for the arm or firm bandage in the leg. The patient is encouraged to rest the nerve as much as possible.

These patients are referred to the senior clinic worker present, for the treatment of neuritis by anti-inflammatory drugs in addition to antileprosy drugs.

Careful reassessment is carried out at the next clinic visit, to evaluate the response to treatment. Hospital referral is often required for proper neuritis care, but the patient is usually unwilling because he is not incapacitated.

(b) Patients with established nerve damage, having anaesthesia and/or muscle paralysis, are given specific advice on the home self-care required to prevent secondary deformities of tissue injury and absorption, and contractures. This may include acceptable skin protection methods and soaking, scraping, oiling for dry, anaesthetic hands and feet, and simple exercises to strengthen weak muscles or prevent skin or joint contractures. The minimum teaching is given, in a practical way when possible, on the patient's priority need. Advice given is recorded, and progress checked at subsequent visits, encouragement or correction being given as appropriate. When ready, the patient is given any further teaching required.

\section{Benefits and problems}

The following observations are based on our experiences in Salur, and in the leprosy control programmes of other Leprosy Mission centres in Andhra Pradesh and Western India, where this systematic approach has also been introduced. They form the basis of a preliminary, subjective evaluation.

\section{BENEFITS}

1 Neuritis has been detected at an early stage in uncomplaining patients, and treated. The proportion of such cases appears higher in more recently established programmes.

2 During assessment, patients have been educated about the early signs and symptoms of nerve damage. Immediate reporting of paraesthesia, or unusual clumsiness or tiredness, in the hands or feet is emphasized. The probable connection between irregular DDS and development of deformity is also stressed. 
In addition, needs other than neuritis may be revealed during the assessment; specific individual education for such needs has been found more effective than general group health education, and the knowledge seems to spread from patient to patient!

3 The relationship between staff and patients has been strengthened during this regular contact, allowing personal problems, or less obvious but 'felt needs' to be discussed. Although repeat assessments are frequently 'negative', they are, therefore, of positive value in case-holding.

4 The physiotherapist has in several instances been transformed from a disillusioned, aimless worker into a purposeful member of the team, with a definite and important role to play.

\section{PROBLEMS}

The frequent occurrence of similar problems in different programmes suggests that there are real difficulties in implementing this approach. They appear to fall into 3 broad, but overlapping categories.

\section{Management problems}

Ideally these assessments should be done by a physiotherapist, whose main role is to minimize deformity. However, reliance on physiotherapy staff exclusively to undertake this care has resulted in no assessments being done in the following situations:

(a) At small clinics, where the presence of a physiotherapist for several hours, in order to see a handful of patients, is not justified; in programmes where domiciliary treatment is given; and in situations where for any other reason a physiotherapist is not allotted to the control programme.

(b) At clinics held concurrently with other clinics in the same programme, when the physiotherapist can only be in one place at a time; unless his tour programme is carefully planned, he will repeatedly see the same patients at a given clinic, whilst others, attending a different week or a different month, are never seen.

(c) When the physiotherapist is on leave, or withdrawn from field activities to cover staff shortages in the 'more urgent' work of the base hospital.

These problems have to do with the priority that is accorded to the early diagnosis, treatment and prevention of deformity; their solution depends on recognition by the whole team of the importance of this activity.

\section{Work organization problems}

(a) The assessment is time consuming at first and in addition there is a 'back-log' of patients to be examined. 
(b) In large clinics it is necessary to select patients most 'at risk' for more frequent rechecking, and to ensure that they do not depart from the clinic unchecked. This applies particularly to irregular attenders, who may be at special risk.

In such conditions, when there is too much work for the physiotherapist, the priorities must be carefully defined, and consideration given to arranging for other staff to undertake part of the work.

\section{Training problems}

Many defects in implementation were due to lack of relevant knowledge and/or practical skills, or failure to adapt and apply these in the field situation; these, therefore, suggest deficiencies in training.

(a) Training of physiotherapists. (i) The training of leprosy physiotherapy staff is usually hospital and surgery orientated, and therefore many workers are afterwards assigned to field programmes with no idea of their role or goals. Specific teaching on the principles of deformity prevention in the field is needed, in addition to the detailed technique of the assessments.

(ii) The assessment is not an end in itself. The physiotherapist must be taught to exercise judgement, and in particular to decide whether, as a result of his assessment, the patient should (a) have routine assessments in the future; (b) have more frequent assessments; (c) have local treatment on the physiotherapist's advice; and/or (d) be referred to senior staff for special treatment.

If each patient is seen as an individual, the physiotherapist will escape the monotony of simple repetitive work and avoid careless mistakes.

(b) Training of more senior staff. At times, the staff responsible have failed to give immediate, and appropriate, neuritis treatment, though indicated. This has arisen from:

(i) Lack of understanding about the purpose of the assessment, and inability to interpret the findings - the assessment is seen as an end in itself, a duty to be performed by the physiotherapist.

(ii) Belief that neuritis always causes the patient to complain-and if not complaining, no treatment is required.

(iii) Unbelief that early nerve damage can be reversed or limited-once demonstrated possible, effort to save nerves increases dramatically.

(iv) Failure to understand that the nerve requires treatment when muscle weakness is recent, and that exercises are not appropriate as the main line of treatment.

It is essential that deficiencies of this sort are corrected by training. A physiotherapist who detects neuritis and gives correct physical treatment and advice to the patient is frustrated if the staff responsible do not prescribe drugs or encourage hospital referral. The fact that silent and mild neuritis, as described 
above, must be taken as seriously as acute neuritis typically associated with reaction, must be emphasized to all, if deformity is to be prevented.

For supervisory purposes, it is also necessary that senior staff know which patients are most likely to develop neuritis and deformity, and so ensure their regular rechecking by the physiotherapist.

(c) Training of more junior staff. Since this approach does not require highly-specialized physiotherapy techniques, it can be learned by most other field workers. Deformity care is already included in the syllabus of training programmes for paramedical workers (non-medical assistants) in India, and in defining the roles of various categories of workers in the National Leprosy Control Programme of India, ${ }^{6}$ Hasan indicates the overlap of responsibility for deformity care and prevention among different workers; though whether detection of silent neuritis is included is uncertain.

\section{Discussion}

This approach requires staff to have basic skills in assessing nerve function, and recording and interpreting results in terms of treatment needed to reverse or minimize nerve damage and therefore prevent deformity. It also needs considerable practical organization if it is to be used effectively on our many patients at widely differing clinics.

Key factors in successful implementation appear to be: 1, a changed emphasis in the teaching of deformity prevention and care to all categories of staff working in the field, not least the physiotherapist; 2, recognition of local variations in programme structure, requiring specific management decisions; and 3, good clinic organization at the local level.

Particular problems may be encountered when staff other than physiotherapists are required to undertake assessments. Our experience suggests that paramedical workers are reluctant to share in the deformity care of their patients, even when having the knowledge, and time, to do it; understandably they feel they are doing the physiotherapist's work in addition to their own duties. But to meet the types of situation listed above, and to ensure the total care of patients at all times, some flexibility is necessary. Appropriate assignment of specific tasks to each member of the control team must be made according to particular local needs, and written into the job description.

Although teaching on deformity care, or 'physiotherapy aspects', is included in training courses for various categories of leprosy staff, our experience suggests that there is need for continuing effort to clarify training objectives, especially with regard to deformity prevention in the field situation. Careful elucidation of students' learning objectives, with theoretical and practical teaching based on these, will help to change the emphasis from care of established deformities to prevention of nerve damage and new deformity, impart the necessary skills and also rectify specific misconceptions. 
If a programme of this sort is to be implemented successfully, careful training, detailed management and positive supervision are required. In addition, however, changes of attitude are often needed, so that staff at all levels are positively motivated to prevent and lessen deformity in their patients. Only the interest, guidance and supervision of the senior team members can achieve this change.

\section{Acknowledgements}

I thank the physiotherapy staff at Salur for their willingness to introduce, adapt, and so refine the approach described here, as well as the leprosy control staff at Salur and other leprosy mission centres in Andhra Pradesh, Karnataka and Maharashtra for their cooperation. I also thank Dr Alexander Thomas (Superintendent, Salur) and Dr R H Thangaraj (Director, The Leprosy Mission, Southern Asia) for their encouragement and permission to write this paper. I am grateful to Dr M E Duncan, Dr J M H Pearson and Miss P J Neville for their help in the preparation of this paper.

\section{References}

1 Wheate HW, Pearson JMH. A Practical Guide to the Diagnosis and Treatment of Leprosy in the Basic Health Unit. ALERT, 1978.

2 Pearson JMH, Ross WF. Nerve involvement in leprosy-pathology, differential diagnosis and principles of management. Lepr Rev, 1975; 46: 199-212.

3 Duncan ME, Pearson JMH. Neuritis in pregnancy and lactation. Int J Lepr, 1982; 50: 31-7.

${ }^{4}$ Srinivasan H, Rao KS, Shanmugam N. Steroid therapy in recent 'quiet nerve paralysis' in leprosy. Lepr India, 1982; 54: 412-19.

${ }^{5}$ Pearson JMH. The evaluation of nerve damage in leprosy. Lepr Rev, 1982; 53: 119-30.

${ }^{6}$ Hasan S. The aims and methods of physiotherapy in field conditions in the National Leprosy Control Programme. Lepr India, 1981; 53: 608-19. 